\title{
The modified integral method for the determination of gravity disturbance near the Earth's surface
}

DOI: https://doi.org/10.1515/jogs-2019-0016

Received November 30, 2018; accepted August 7, 2019

\begin{abstract}
For the calculation of gravity disturbance in the Earth's external gravity field, the Stokes-Pizzetti integral is a commonly used method. However, when the target point approaches the Earth's surface, such problems as singularity and discontinuity arise due to the Stokes kernel structure itself. To settle the problems, firstly the reason for singularity and discontinuity was discussed, and then modification was made to the integral formula, by which the singularity at the surface point is eliminated. Finally the nonsingular integral formulas for the calculation of disturbing gravity were derived. In numerical experiments, an area in China was selected to test the modified formula. Numerical results show that the modified formula performs much better than classical Stokes-Pizzetti integral formula when dealing with the calculation of the radial component of gravity disturbance near the Earth's surface.
\end{abstract}

Keywords: discontinuity; radial component of gravity disturbance; Stokes integral kernel; singularity

\section{Introduction}

On the basis of the classical Stokes theory and Molodensky theory, a lot of wide and deep research has been made on the calculation methods of the elements of the Earth's external gravity field. The methods could be divided into two kinds: model approximation and algorithm approximation. The former includes the Stokes integral method, upward continuation method, etc., and those proposed in modern physical geodesy, like Molodensky solution, Brovar solution and analytical continuation solution. The

\footnotetext{
*Corresponding Author: D. Zhao: Surveying and Mapping Engineering Department, Geospatial Information College, Information Engineering University, Zhengzhou Science Avenue 1, Henan Province, China, 450001, E-Mail: zhaodongming0510@126.com

S. Li, Q. Wang, Z. Gong: Surveying and Mapping Engineering Department, Geospatial Information College, Information Engineering University, Zhengzhou Science Avenue 1, Henan Province, China, 450001
}

algorithm approximation methods include the Bjerhammar solution, single layer density solution, point mass solution, least square collocation solution and spherical harmonics solution.

The Stokes integral method provides the external disturbing potential solution based on the boundary value problem of spherical gravity anomaly. (Heiskanen WA, 1967) described the principle for the calculation of gravity disturbance using the Stokes integral, along with the derivation of the formulas of the components of gravity disturbance. (Featherstone WE, 2003) proposed five modification methods of the Stokes integral kernel, and he made comparisons and analyses on truncation error and accuracy of the modified integration kernel using Australian gravity observations. (Najafi-Alamdari, 2006) derived two types of ellipsoidal correction formulae of the Stokes integration kernel. The Stokes integral is further applied along with the remove-and-restore technique (Yildiz et al, 2012).

The upward continuation method belongs to the first kind boundary value problem. In the method the Poisson integral is applied in the derivation of the external disturbing gravity potential using the gravity potential on the Earth's surface.

The Molodensky solution is based on the boundary value problem on the telluroid, from which the anomalous potential is represented as a single layer potential on the telluroid, and the gravity disturbance is calculated by converting the boundary conditions into integral equations. Based on the Molodensky theory, the Brovar solution also provides the gravity disturbance by solving the integral equation, and when the telluroid coincides with the sphere, the integral term in the integral equation becomes zero and thus the computation is simplified. As for the analytical solution, the surface gravity anomaly is continued onto the level surface of the computation point, and then the gravity disturbance is calculated using the Stokes integral. (Moritz H, 1979) presented in detail the basics and formulas for the above solutions. (Tziavos, 2013) analyzed the role the terrain correction term plays in the analytical continuation solution to Molodensky problem. (Sanso, 
2017) proved the equivalence between the analytical solution and Bjerhammar solution and pointed out the difference in connotation between the two solutions.

(Hsu H, 1984) proposed the single layer density method from Bjerhammar theory for the computation of the external disturbing potential using virtual single layer density, which simplifies the integral kernel's structure. (Bjerhammar, 1987) presented the solution to the Bjerhammar's virtual sphere boundary value problem with the single layer density being parameter. (Sideris, 1986) discussed the use of FFT and therefore improves the calculation speed dramatically. As the discretization form of single layer density method, point mass method was more widely used in computations. (Rüdiger L, 1993) proposed the free-positioned point mass method, which is more flexible, and (Miao L, 2014) described the four important parameters and their computations of the free-positioned point mass method. The point mass method has been discussed in literature on regional gravity field modeling (Denker, 2013) due to its kernel's structure.

In the paper the main topic is focused on the singularity and discontinuity problem of the Stokes integral method in the calculation of gravity disturbance components when the computation point approaches or even coincides with the mobile point on the boundary surface. The paper aims at the improvement of the integral kernel in order to reduce the singularity and discontinuity problem in the computation of gravity disturbance when the computation point approaches the boundary surface from outside.

\section{The integral method of the determination of external disturbing gravity potential based on Molodensky Problem}

In the classical Stokes theory, the solution to the external disturbing potential $T$ is founded on the sphere approximation of the Earth's surface, however the Molodensky theory deals with the following boundary value problem

$$
\left.\begin{array}{l}
\Delta T(P)=0, P \text { outside } \Sigma \\
{\left[-\frac{\partial T}{\partial \rho}-\frac{2 T}{\rho}\right]_{\Sigma}=\Delta g(\Sigma)} \\
T \rightarrow 0, \text { when } \rho \rightarrow \infty
\end{array}\right\}
$$

The variables and symbols are illustrated in Fig. 1 and Fig. 2, where $\Sigma$ is the natural surface of the Earth, and on the surface we have ground gravity anomalies $\Delta g(\Sigma)$. $(\lambda, \varphi, \rho)$ is the spherical coordinates of the computation point $P$, in which $\lambda$ is longitude, $\varphi$ is geocentric latitude and $\rho$ is geocentric distance. When viewing the Earth's surface as a sphere with radius $R$, the external disturbing potential $T$ can be formulated by Eq. (2) using the StokesPizzetti integral

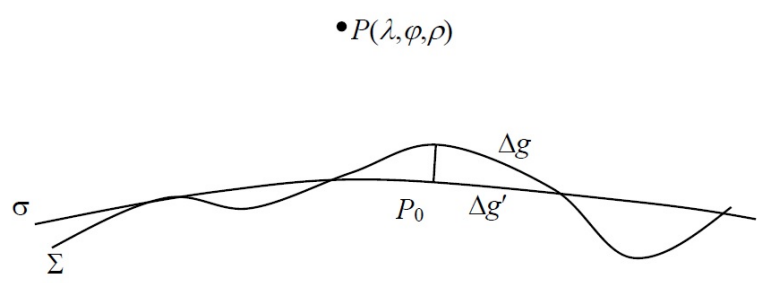

Fig. 1. Boundary surface of Molodensky Problem

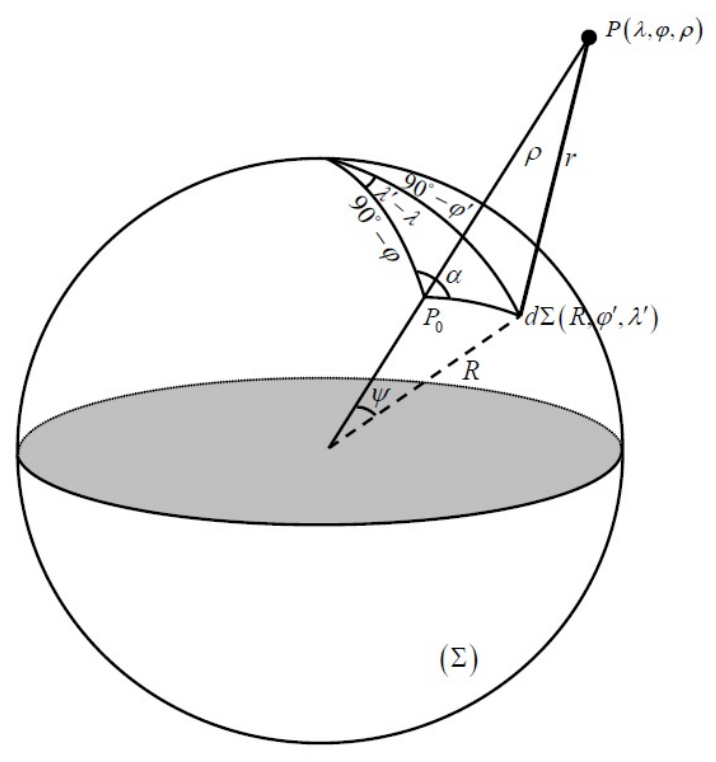

Fig. 2. Variables in the Stokes kernel

$$
T(\lambda, \varphi, \rho)=\frac{1}{4 \pi} \iint_{\Sigma} \Delta g(\Sigma) S(\rho, \psi) d \Sigma
$$

$S(\rho, \psi)$ is the kernel function. $d \Sigma$ is the spherical unit element, $d \Sigma=R^{2} \cos \varphi^{\prime} \mathrm{d} \varphi^{\prime} \mathrm{d} \lambda^{\prime} . \psi$ is the spherical angle between the two radius of the computation point and the mobile point on the sphere.

As the spherical approximation of the Earth's surface will result in deviations for terrain such as mountain area, in that case the ground gravity anomaly $\Delta g$ can be analytically continued to some level surface $\sigma$ (Moritz, 1980). Denote the gravity anomaly on the level surface as $\Delta g^{\prime}$, and 
then the external disturbing potential $T$ can be formulated as follows

$$
T(\lambda, \varphi, \rho)=\frac{1}{4 \pi} \iint_{\sigma} \Delta g^{\prime} S(\rho, \psi) d \sigma
$$

\section{The discontinuity and singularity problem of the integral method near the Earth's surface}

(Heiskanen WA, 1967) presents the generalized Stokes formula that takes into the 0 -th term

$$
T(\lambda, \varphi, \rho)=\frac{1}{4 \pi} \iint_{\Sigma} \Delta g\left(S(\rho, \psi)-\frac{1}{\rho}\right) d \Sigma
$$

Take the notation

$$
\bar{S}(\rho, \psi)=S(\rho, \psi)-\frac{1}{\rho}
$$

and the three components of gravity disturbance can be formulated as follows

$$
\begin{aligned}
\delta_{\rho}= & \frac{1}{4 \pi} \iint_{\Sigma} \Delta g(\Sigma) \frac{\partial \bar{S}(\rho, \psi)}{\partial \rho} d \Sigma \\
\delta_{\lambda}= & -\frac{1}{4 \pi \rho} \iint_{\Sigma} \Delta g(\Sigma) \frac{\partial \bar{S}(\rho, \psi)}{\partial \rho} \sin \alpha d \Sigma \\
\delta_{\varphi}= & -\frac{1}{4 \pi \rho} \iint_{\Sigma} \Delta g(\Sigma) \frac{\partial \bar{S}(\rho, \psi)}{\partial \rho} \cos \alpha d \Sigma \\
\frac{\partial \bar{S}(\rho, \psi)}{\partial \rho}= & -\frac{2(\rho-R \cos \psi)}{r^{3}}-\frac{3}{\rho r}+\frac{6 r}{\rho^{3}}+\frac{13 R}{\rho^{3}} \cos \psi \\
& +\frac{6 R}{\rho^{3}} \cos \psi \ln \frac{\rho-R \cos \psi+r}{2 \rho} \\
\frac{\partial \bar{S}(\rho, \psi)}{\partial \psi}= & -\frac{2 \rho R \sin \psi}{r^{3}}-\frac{3 R \sin \psi}{\rho r}+\frac{5 R \sin \psi}{\rho^{2}} \\
& +\frac{3 R \sin \psi}{\rho^{2}} \ln \frac{r+\rho-R \cos \psi}{2 \rho} \\
& -\frac{3 R^{2}(\rho+r) \sin \psi \cos \psi}{\rho^{2} r(r+\rho-R \cos \psi)}
\end{aligned}
$$

From Figure 2, $r$ is the distance from the computation point to the mobile point on the boundary surface, and therefore when $r$ is equal to 0 or near 0 , then singularity problem will arise for Eq. (6) (8).

On the other hand, take the notation $\bar{S}(\rho, \psi)=\frac{2}{r}+$ $Q(\rho, \psi)$, and then Eq. (4) can be expressed as

$$
T(P)=\frac{1}{4 \pi} \iint_{\Sigma}\left[\frac{2}{r}+Q(\rho, \psi)\right] \Delta g d \Sigma
$$

where

$$
Q(\rho, \psi)=-\frac{3 r}{\rho^{2}}-\frac{5 R \cos \psi}{\rho^{2}}-\frac{3 R}{\rho^{2}} \cos \psi \ln \frac{r+\rho-R \cos \psi}{2 \rho}
$$

Denote the two integrals on the right of Eq.(9) as $T_{1}$ and $T_{2}$,

$$
\begin{aligned}
& T_{1}=\frac{1}{4 \pi} \iint_{\sigma} \frac{2}{r} \Delta g d \Sigma \\
& T_{2}=\frac{1}{4 \pi} \iint_{\sigma} \Delta g Q(\rho, \psi) d \Sigma
\end{aligned}
$$

According to (Heiskanen and Moritz, 1967), when the calculation point $P$ approaches the surface layer, the derivative of $T_{1}$ with respect to radius is similar to that of a single layer potential

$$
\left(\frac{\partial T_{1}}{\partial \rho}\right)_{P \rightarrow P_{0}}=-\Delta g+\frac{1}{4 \pi} \iint_{\Sigma} \Delta g \frac{\partial}{\partial \rho}\left(\frac{2}{r}\right) d \Sigma
$$

From Eq.(9) the radial component of the gravity disturbance outside the boundary surface has the following expression

$$
\begin{aligned}
\delta_{\rho}(P)= & \frac{1}{4 \pi} \iint_{\Sigma} \Delta g(\Sigma) \frac{\partial}{\partial \rho}\left(\frac{2}{r}\right) d \Sigma \\
& +\frac{1}{4 \pi} \iint_{\Sigma} \Delta g(\Sigma) \frac{\partial Q(\rho, \psi)}{\partial \rho} d \Sigma
\end{aligned}
$$

And when the computation point passes the surface of boundary, the limit of the radial component of gravity disturbance is

$$
\begin{aligned}
\delta_{\rho}(P)_{P \rightarrow P_{0}}= & -\Delta g+\frac{1}{4 \pi} \iint_{\Sigma} \Delta g(\Sigma) \frac{\partial}{\partial \rho}\left(\frac{2}{r}\right) d \Sigma \\
& +\frac{1}{4 \pi} \iint_{\Sigma} \Delta g(\Sigma) \frac{\partial Q(\rho, \psi)}{\partial \rho} d \Sigma
\end{aligned}
$$

Comparing Eq. (12) and Eq. (13), the radial component of gravity disturbance is not continuous when the computation point passes the boundary surface from outside, which results in the discontinuity problem.

\section{Improvement of the Stokes-Pizzetti Integral formula for the calculation of gravity disturbance}

Using the orthogonal characteristic of spherical harmonics we have $=0$, and denote the gravity anomaly at the 
spherical point $P_{0}$ as $\Delta g_{P_{0}}$, and we have

$$
\frac{1}{4 \pi} \iint_{\Sigma} \bar{S}(\rho, \psi) \Delta g_{P_{0}} d \Sigma=-\frac{R^{2}}{\rho} \Delta g_{P_{0}}
$$

Substitute Eq. (14) into Eq. (4)

$$
T(P)=\frac{1}{4 \pi} \iint_{\Sigma} \bar{S}(\rho, \psi)\left[\Delta g(\Sigma)-\Delta g_{P_{0}}\right] d \Sigma+\frac{R^{2}}{\rho} \Delta g_{P_{0}}
$$

Considering Eq. (9)

$$
\begin{aligned}
T(P)= & \frac{1}{4 \pi} \iint_{\sigma}\left[\frac{2}{r}+Q(\rho, \psi)\right]\left[\Delta g(\Sigma)-\Delta g_{P_{0}}\right] d \Sigma+\frac{R^{2}}{\rho} \Delta g_{P_{0}} \\
= & \frac{1}{4 \pi} \iint_{\sigma}\left[\Delta g(\Sigma)-\Delta g_{P_{0}}\right] \frac{2}{r} d \Sigma \\
& +\frac{1}{4 \pi} \iint_{\sigma}\left[\Delta g(\Sigma)-\Delta g_{P_{0}}\right] Q(\rho, \psi) d \Sigma+\frac{R^{2}}{\rho} \Delta g_{P_{0}}
\end{aligned}
$$

The first term of the right part of Eq.(16) is the potential generated by a single layer, and it could be proved that its radial derivative is continuous when the computation point passes the sphere

$$
\begin{aligned}
& \left.\frac{1}{4 \pi} \iint_{\Sigma}\left[\Delta g(\Sigma)-\Delta g_{P_{0}}\right] \frac{\partial}{\partial \rho}\left(\frac{2}{r}\right) d \Sigma\right|_{P \rightarrow P_{0}} \\
& =\left[\Delta g(\Sigma)-\Delta g_{P_{0}}\right]_{P \rightarrow P_{0}}+\frac{1}{4 \pi} \iint_{\Sigma}\left[\Delta g(\Sigma)-\Delta g_{P_{0}}\right] \frac{\partial}{\partial \rho}\left(\frac{2}{r}\right) d
\end{aligned}
$$

Because $\left[\Delta g(\Sigma)-\Delta g_{P_{0}}\right]_{P=P_{0}}=0$, from Eq. (17) we have

$$
\begin{aligned}
& \left.\frac{1}{4 \pi} \iint_{\Sigma}\left[\Delta g(\Sigma)-\Delta g_{P_{0}}\right] \frac{\partial}{\partial \rho}\left(\frac{2}{r}\right) d \Sigma\right|_{P \rightarrow P_{0}} \\
& =\frac{1}{4 \pi} \iint_{\Sigma}\left[\Delta g(\Sigma)-\Delta g_{P_{0}}\right] \frac{\partial}{\partial \rho}\left(\frac{2}{r}\right) d \Sigma
\end{aligned}
$$

Therefore we have

$$
\delta_{\rho}(P)=\frac{1}{4 \pi} \iint_{\Sigma}\left[\Delta g(\Sigma)-\Delta g_{P_{0}}\right] \frac{\partial \bar{S}(\rho, \psi)}{\partial \rho} d \Sigma-\frac{R^{2}}{\rho^{2}} \Delta g_{P_{0}}
$$

Eq. (18) is continuous from the boundary surface to the exterior space due to the fact that $\left(\Delta g-\Delta g_{P_{0}}\right)$ is zero on the surface.

For the horizontal components of disturbing gravity we have

$$
\begin{aligned}
\delta_{\varphi}(P) & =-\frac{1}{4 \pi \rho} \iint_{\Sigma} \Delta g \frac{\partial \bar{S}(\rho, \psi)}{\partial \psi} \cos \alpha d \Sigma \\
& =-\frac{1}{4 \pi \rho} \iint_{\Sigma}\left[\Delta g(\Sigma)-\Delta g_{P_{0}}\right] \frac{\partial \bar{S}(\rho, \psi)}{\partial \psi} \cos \alpha d \Sigma
\end{aligned}
$$

$$
\begin{aligned}
\delta_{\lambda}(P) & =-\frac{1}{4 \pi \rho} \iint_{\Sigma} \Delta g \frac{\partial \bar{S}(\rho, \psi)}{\partial \psi} \sin \alpha d \Sigma \\
& =-\frac{1}{4 \pi \rho} \iint_{\Sigma}\left[\Delta g(\Sigma)-\Delta g_{P_{0}}\right] \frac{\partial \bar{S}(\rho, \psi)}{\partial \psi} \sin \alpha d \Sigma
\end{aligned}
$$

\section{Numerical experiments}

For the test of the proposed method, a close-loop numerical experiment on the calculation of gravity disturbance was made. An area of $2^{\circ} \times 2^{\circ}$ coverage in China was selected and the computation points are located in the grids of different altitudes with $2^{\prime} \times 2^{\prime}$ resolution above the selected area, and therefore, the number of computation points are the same for each height level.

Firstly, the three gravity disturbance components of each computation point were calculated using the EIGEN6C4 gravity field model which is truncated to degree and order 2160, which are taken as reference values or true values. The unit of the value of gravity disturbance is mgal (1mgal $\left.=10^{-5} \mathrm{~ms}^{-2}\right)$.

Secondly, to calculate the gravity disturbances of grid points of different altitudes using the classical Stokes inte$\Sigma$ gral and the proposed method of the paper, ground gravity anomalies of $10^{\circ} \times 10^{\circ}$ coverage were computed using the EIGEN-6C4 of $2160 \mathrm{~d} / \mathrm{o}$ and then gravity anomalies of $2^{\prime} \times 2^{\prime}$ resolution were generated using interpolation process, due to the fact that the EIGEN-6C4 model is equivalent to $5^{\prime} \times 5^{\prime}$ resolution. Based on the generated gravity anomalies, the gravity disturbance of the computation points were calculated using the classical Stokes integral and modified Stokes integral method respectively.

For the computation points within different altitude grids, there are two kinds of gravity disturbance difference: true values vs classical Stokes integral results, and true values vs modified Stokes integral method. Statistics were made based on the gravity disturbance difference for each height level, which are shown in Figure 3 and Figure 4, where $\delta g_{\lambda}, \delta g_{\phi}, \delta g_{r}$ represents the longitudinal, latitudinal and radial component of gravity disturbance respectively. In the following figures, std is the short for standard deviation.

Comparing Fig. 3 and Fig. 4, Fig. 5 and Fig. 7, Fig. 6 and Fig. 8, we can find that the stds of $\delta g_{r}$ component difference between classical Stokes integral values and true values vary dramatically from near the surface to the altitude $10 \mathrm{~km}$ above the surface, and the stds of $\delta g_{r}$ component difference between modified Stokes integral values and true values vary relatively smoother, which indicates a 


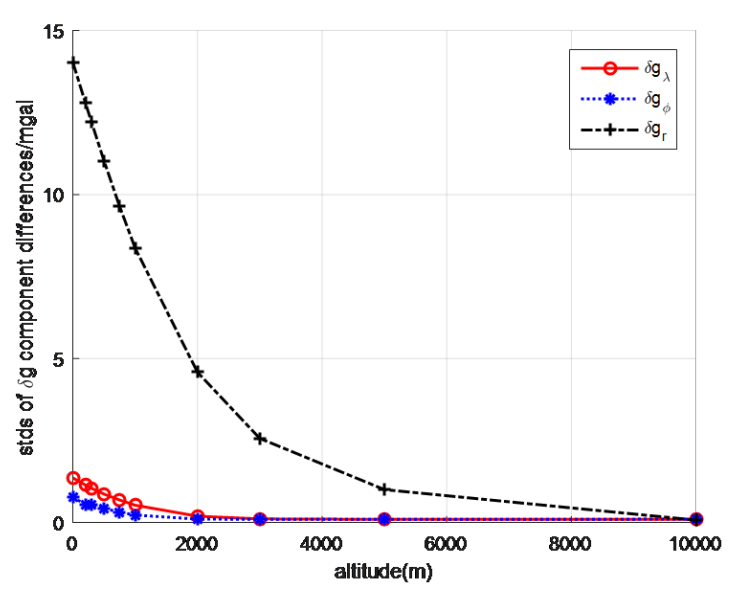

Fig. 3. stds of $\delta g$ component difference between classical Stokes integral values and true values

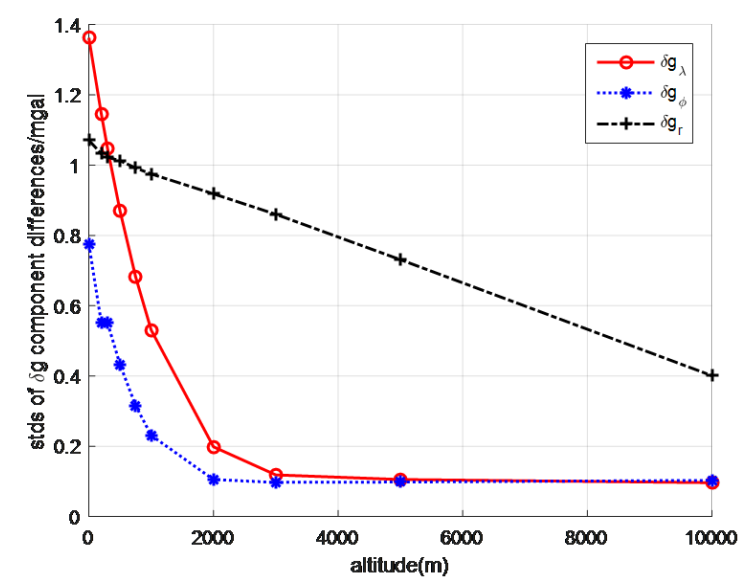

Fig. 4. stds of $\delta g$ component difference between classical Stokes integral values and true values

stable accuracy, from near the surface to the altitude $10 \mathrm{~km}$ above the surface.

\section{Summary}

In the calculation of external disturbing gravity, the classical Stokes-Pizzetti integral is widely applied. However, it has such defects as singularity and discontinuity problem when the calculation point approaches the boundary surface very closely. The singularity problem is caused by the near 0 distance between the computation point and the mobile point on the surface and when the computation point approaches the surface, the first-order derivative of disturbing potential with respect to normal is not continuous. In the paper, the reason for the singularity problem

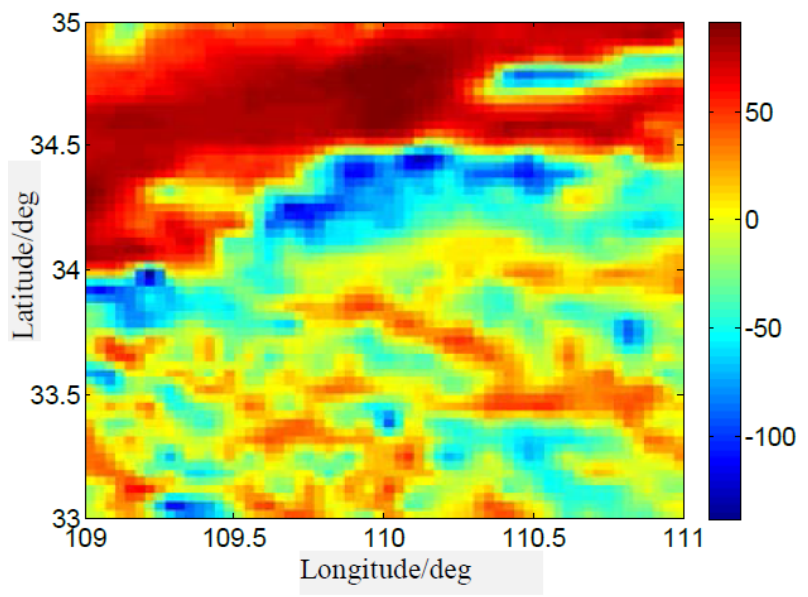

Fig. 5. difference of $\delta g_{r}$ between classical Stokes integral values and true values at altitude near $0 \mathrm{~m}$

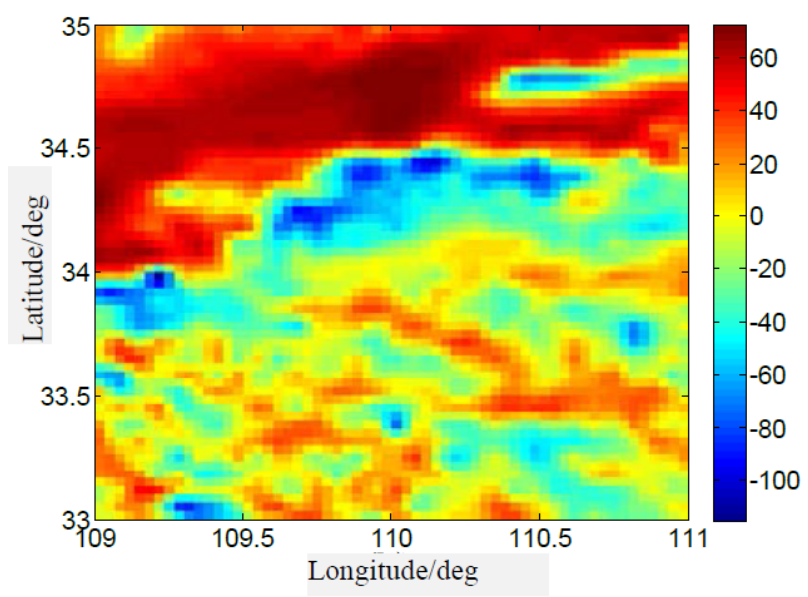

Fig. 6. difference of $\delta g_{r}$ between classical Stokes integral values and true values at altitude $500 \mathrm{~m}$

and discontinuity problem were theoretically illustrated by formulation.

The modified Stokes-Pizzetti integral formula was furthermore proposed. The proposed modified StokesPizzetti integral formula is not only continuous when approximating the surface, but also has no singularity problem. Numerical results show that the modified formula performs much better than classical Stokes-Pizzetti integral formula when dealing with the calculation of the radial component of gravity disturbance near the Earth's surface. Numerical results also show that both the classical Stokes-Pizzetti integral and the modified Stokes integral have almost the consistent accuracy when used to calculate the horizontal components of gravity disturbance. It is recommended that the modified Stokes integral be used to calculate the gravity disturbance near the Earth's surface. 


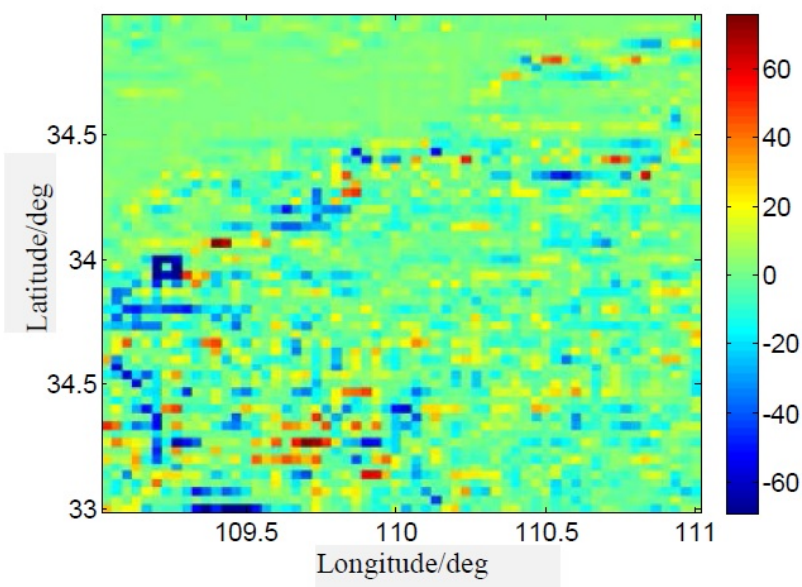

Fig. 7. difference of $\delta g_{r}$ between modified Stokes integral values and true values at altitude near $0 \mathrm{~m}$

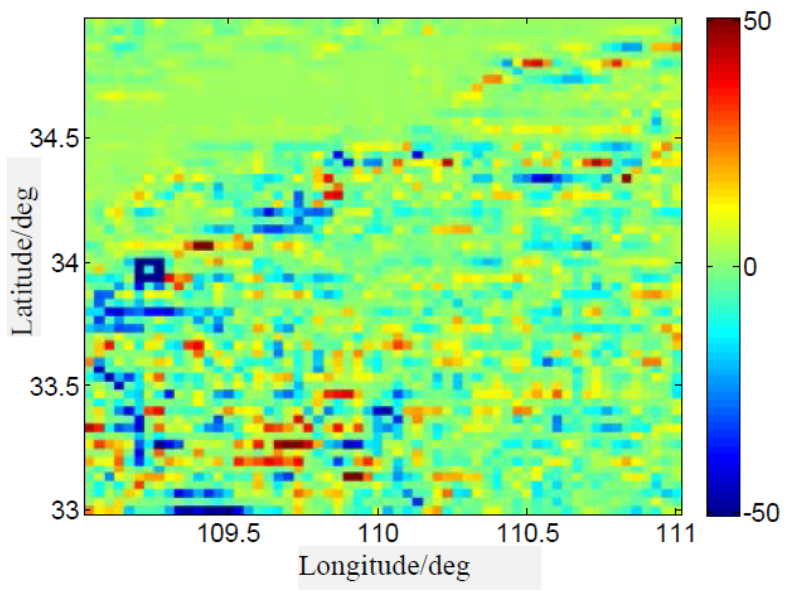

Fig. 8. difference of $\delta g_{r}$ between modified Stokes integral values and true values at altitude $500 \mathrm{~m}$

Acknowledgements: The work in the paper is financially supported by the Chinese National Natural Science Foundation project No.41574020.

\section{References}

Amos MJ and Featherstone WE, Comparisons of recent global geopotential models with terrestrial gravity field data over New Zealandand Australia. Geomatics Res Australas, 2003, 79,1-20

Bjerhammar A., Discrete Physical Geodesy. Rep No. 380, Dept. of Geodetic Science and Surveying, the Ohio State University, Columbus, 1987

Denker H., Regional gravity field modeling: Theory and Practical Results. In: Xu G.C. (Ed.), Science of Geodesy - II. Springer Verlag, Heidelberg, Germany, 2013, 185-291.
Goli M., Najafi-Alamdari M and Vaníček P. Numerical behaviour of the downward continuation of gravity anomalies. Studia Geophysica et Geodaetica, 2011, 55(2): 191-202.

Heiskanen,W.A., and Moritz., Physical Geodesy , W.H.Freeman, San Francisco, 1967

Hsu Houtze and Zhu Zhuowen. The virtual single layer density representation of the Earth's external gravity field. China Science, 1984, 6, 93-98.

Kloch G.and Krynski J., On the Determination of the Terrain Correction Using the Spherical Approach. In: Mertikas S. (eds) Gravity, Geoid and Earth Observation. International Association of Geodesy Symposia, 2010, vol 135. Springer, Berlin, Heidelberg

Lin, M., Denker, H. and Müller, J. Regional gravity field modeling using free-positioned point masses. Stud Geophys Geod, 2014, 58, 207. https://doi.org/10.1007/s11200-013-1145-7

M. Najafi-Alamdari and S. R. Emadi; K. Moghtased-Azar, The ellipsoidal correction to the Stokes kernel for precise geoid determination, J Geod, 2006, 80, 675-689

Moritz, Advanced Physical Geodesy, Herbert Wichmann Verlag, Karlsruhe, 1979

Moritz, H., Linear Solutions of the Geodetic Boundary-Value Problem, Dept. of Geodetic Science and Surveying, the Ohio State University, Columbus, 1966

Rüdiger L. The method of free-positioned point masses - geoid studies on the Gulf of Bothnia Bulletin Geodesique, 1993, 67: 31. https://doi.org/10.1007/BF00807295

Sansò F.and Sideris M.G., On the Equivalent BVPs of Stokes and Helmert, and Their Relations to the Molodensky BVP by Analytical Continuation. In: Geodetic Boundary Value Problem: the Equivalence between Molodensky's and Helmert's Solutions. SpringerBriefs in Earth Sciences. Springer, Cham, 2017

Sideris, M.G. and Schwarz, K.P. Solving Molodensky's series by fast Fourier transform techniques Bull. Géodésique, 1986, 60: 51. https://doi.org/10.1007/BF02519354

Tscherning C.C., Comparison of some methods for the detailed representation of the Earth's gravity field. Rev. Geophys., 1981, $19,213-221$.

Tziavos I.N. and Sideris M.G., Topographic Reductions in Gravity and Geoid Modeling. In: Sansó F., Sideris M. (eds) Geoid Determination. Lecture Notes in Earth System Sciences, 2013, vol 110. Springer, Berlin, Heidelberg

Yildiz H, Forsberg R, Agren J, Tscherning C and Sjöberg L., Comparison of remove-compute-restore and least squares modification of Stokes' formula techniques to quasi-geoid determination over the Auvergne test area. Journal of Geodetic Science, 2012, 2, 53-64. 